\title{
Entanglement entropy in low-energy field theories at a finite chemical potential
}

\author{
Ivan Morera, ${ }^{1,2}$ Irénée Frérot, ${ }^{3,4}$ Artur Polls, ${ }^{1,2}$ and Bruno Juliá-Díaz ${ }^{1,2,3}$ \\ ${ }^{1}$ Departament de Física Quàntica i Astrofísica, Facultat de Física, Universitat de Barcelona, E-08028 Barcelona, Spain \\ ${ }^{2}$ Institut de Ciències del Cosmos, Universitat de Barcelona, ICCUB, Martí i Franquès 1, Barcelona 08028, Spain \\ ${ }^{3}$ ICFO-Institut de Ciencies Fotoniques, The Barcelona Institute of Science and Technology, 08860 Castelldefels (Barcelona), Spain \\ ${ }^{4}$ Max-Planck-Institut für Quantenoptik, D-85748 Garching, Germany
}

(Received 19 April 2020; accepted 12 June 2020; published 2 July 2020)

\begin{abstract}
We investigate the leading area-law contribution to entanglement entropy in a system described by a general Lagrangian with $O(2)$ symmetry containing first- and second-order time derivatives, namely, breaking the Lorentz invariance. We establish a connection between the Higgs gap present in a symmetry-broken phase and the area-law term for the entanglement entropy in the general nonrelativistic case. Our predictions for the entanglement entropy and correlation length are successfully compared to numerical results in two paradigmatic systems: the Mott insulator to the superfluid transition for ultracold lattice bosons and the ground state of ferrimagnetic systems.
\end{abstract}

DOI: 10.1103/PhysRevResearch.2.033016

\section{INTRODUCTION}

In condensed-matter physics, relativistic quantum field theories often arise as low-energy effective approximations. However, in multiple situations, the local Lorentz invariance is lost. As a prominent example, near a quantum phase transition [1], a dynamical critical exponent different from one indicates the different scaling of space and time. A second example is non-Lorentz-invariant systems where the ground state spontaneously breaks a symmetry of the Hamiltonian. Goldstone's theorem ensures the presence of Nambu-Goldstone (NG) bosons at low energy [2,3], but the lack of Lorentz invariance may dramatically change their dispersion relation [4-7]. Nonrelativistic NG bosons have been extensively studied recently [7] and naturally appear in the many-body context [8], e.g., in the presence of long-range interactions [9].

The nature of the low-energy excitations of a many-body system is deeply related to the quantum fluctuations in the ground state and has a profound impact on the structure of quantum entanglement across the system. For instance, the scaling of ground-state entanglement entropy with the subsystem size displays a logarithmic violation of the socalled area law [10-15] in one-dimensional gapless systems with short-range interactions [13] or in the presence of a Fermi surface in any dimension [16,17], and, in bosonic systems with spontaneous symmetry breaking, a subdominant universal additive logarithmic correction carries the nature of the Goldstone modes [9,18,19].

In the present paper, we study the entanglement present in a general nonrelativistic field-theoretical description with

Published by the American Physical Society under the terms of the Creative Commons Attribution 4.0 International license. Further distribution of this work must maintain attribution to the author(s) and the published article's title, journal citation, and DOI.
$O(2)$ symmetry. The latter appears both in many condensedmatter-physics phenomena [1] and in particle physics at a finite chemical potential, e.g., kaon condensation [20] with an enlarged $U(2)$ symmetry. We show that the dominant arealaw prefactor of entanglement entropy acquires a universal contribution throughout the phase diagram, associated with the finite correlation length in the gapped disordered phase, and to an elusive "Higgs correlation length" in the gapless ordered phase, associated with amplitude fluctuations of the order parameter. We discuss the relevance of our analytical field-theory predictions for two prominent examples of many-body phenomena: the superfluid (SF) to Mott insulator (MI) quantum phase transition for ultracold lattice bosons and ferrimagnets, which are gapless yet short-range-correlated systems.

\section{NONRELATIVISTIC LOW-ENERGY THEORY}

We consider the general $O(2)$-invariant Lagrangian density $\mathcal{L}$ describing the dynamics of a complex field $\psi(\vec{r}, t)=\left(\phi_{1}+\right.$ $\left.i \phi_{2}\right) / \sqrt{2}$ in $D+1$ dimensions,

$$
\mathcal{L} / K=\left|\left(\partial_{t}-i \mu_{r}\right) \psi\right|^{2}-c^{2}|\nabla \psi|^{2}-m^{2}|\psi|^{2}-c_{4}|\psi|^{4},
$$

where the global factor $K$ plays the role of a kinetic mass for the field degrees of freedom [see Eq. (2)]. The (relativistic) chemical potential $\mu_{r}$ breaks the Lorentz invariance of the theory and is relevant to many condensed-matter problems where Lorentz invariance is absent [1,21]. For certain systems, such as superconductors, the equations of motion have to be symmetric under complex conjugation as a consequence of particle-hole symmetry [22,23]. This imposes the coefficient of the first-order time derivative to vanish, and, therefore, $\mu_{r}=0$. On the contrary, in pure nonrelativistic systems, the dynamics is driven by a Schrödinger equation and only contains first-order time derivatives, e.g., superfluid helium. The Lagrangian Eq. (1) was studied in the context of relativistic Bose-Einstein condensates [24,25] and more recently in the 
study of nonrelativistic NG bosons [6,7,26], which naturally appear in systems at a finite chemical potential [27-29] where the interplay between first- and second-order time derivatives can lead to the appearance of massive NG bosons [30].

Our purpose is to study the ground-state bipartite entanglement entropy for systems effectively described at low energy by the Lagrangian Eq. (1). In particular, we will consider the influence of the Lorentz-invariance-breaking chemical potential, especially at the quantum phase transition between the disordered phase and the long-range-ordered phase. We consider a subsystem $A$ immersed in an infinite ground state and compute the von Neumann entropy $S$ of its reduced state $\rho_{A}=\operatorname{Tr}_{B}\left|\Psi_{0}\right\rangle\left\langle\Psi_{0}\right|$, where $\operatorname{Tr}_{B}$ denotes the trace over $B$ degrees of freedom (the complement of $A$ ), and $\left|\Psi_{0}\right\rangle$ is the ground state. In $D=2,3, S$ obeys a so-called area law over the whole phase diagram, namely, it scales as $S=a \mathcal{A}$ up to subdominant corrections, where $\mathcal{A}$ is the area of the boundary between the $A$ and the $B$ regions [15]. In gapless $D=1$ systems, $S$ may display a logarithmic violation of the area law, namely, $S \propto \ln L$ where $L$ is the length of the $A$ subsystem [13]. Furthermore, throughout the paper, we will not consider the subdominant corrections [19] and focus, instead, on the area-law coefficient $a$. Our main purpose is to identify universal contributions to $a$.

For our purpose, it is more convenient to work with the Hamiltonian formulation of the theory. We introduce the canonical moments $\pi_{1 / 2}=\frac{\delta \mathcal{L}}{\delta\left(\partial_{t} \phi_{1 / 2}\right)}=K\left(\partial_{t} \phi_{1 / 2} \pm \mu_{r} \phi_{2 / 1}\right)$. The Hamiltonian operator reads in Fourier space,

$$
\begin{aligned}
\mathcal{H}(\mathbf{k})= & \frac{1}{2 K}\left(\pi_{1}^{2}+\pi_{2}^{2}\right)+\frac{K}{2}\left(m^{2}+c^{2} k^{2}\right)\left(\phi_{1}^{2}+\phi_{2}^{2}\right) \\
& +\mu_{r}\left(\phi_{1} \pi_{2}-\phi_{2} \pi_{1}\right)+\frac{K}{4} c_{4}\left(\phi_{1}^{2}+\phi_{2}^{2}\right)^{2},
\end{aligned}
$$

where $\left[\phi_{a}(\mathbf{k}), \pi_{b}\left(\mathbf{k}^{\prime}\right)\right]=i \delta_{a, b} \delta\left(\mathbf{k}-\mathbf{k}^{\prime}\right)$ and $\left[\phi_{a}(\mathbf{k}), \phi_{b}\left(\mathbf{k}^{\prime}\right)\right]=$ $\left[\pi_{a}(\mathbf{k}), \pi_{b}\left(\mathbf{k}^{\prime}\right)\right]=0$. Throughout the paper, we will consider a Gaussian approximation to the ground state, accounting for harmonic quantum fluctuations around a saddle-point (mean-field) solution. Our focus is the entanglement content of such quantum fluctuations. At the mean-field level, two phases are found: a disordered phase for $m^{2} \geqslant \mu_{r}^{2} \quad$ (such that $\phi_{1}^{(0)}=\phi_{2}^{(0)}=\pi_{1}^{(0)}=\pi_{2}^{(0)}=0$ ) and an ordered phase for $m^{2}<\mu_{r}^{2}$ where the $O(2)$ symmetry is spontaneously broken $\left[\phi_{2}^{(0)}=\pi_{1}^{(0)}=\right.$ $0, \phi_{1}^{(0)}=\sqrt{\left(\mu_{r}^{2}-m^{2}\right) / c_{4}}, \pi_{2}^{(0)}=-K \mu_{r} \phi_{1}^{(0)}$ e.g., $\quad \phi_{2}^{(0)}=$ $\left.\pi_{1}^{(0)}=0, \phi_{1}^{(0)}=\sqrt{\left(\mu_{r}^{2}-m^{2}\right) / c_{4}}, \pi_{2}^{(0)}=-K \mu_{r} \phi_{1}^{(0)}\right]$. The quadratic Hamiltonian governing the harmonic fluctuations around this mean-field solution is found upon replacing $\phi_{1 / 2} \rightarrow \phi_{1 / 2}^{(0)}+\phi_{1 / 2}$ and $\pi_{1 / 2} \rightarrow \pi_{1 / 2}^{(0)}+\pi_{1 / 2}$ and neglecting terms of order $\mathcal{O}\left(\phi_{1 / 2}^{3}, \pi_{1 / 2}^{3}\right)$ and higher. We also subtract the mean-field ground-state energy contribution.

\section{DISORDERED PHASE}

In the disordered phase $\left(m^{2} \geqslant \mu_{r}^{2}\right)$, the quadratic Hamiltonian is simply obtained from Eq. (2) by setting $c_{4}=0$. The excitation spectrum displays two gapped modes of energy $\omega_{\mathbf{k}}^{ \pm}=\Omega_{\mathbf{k}} \pm \mu_{r}$ with $\Omega_{\mathbf{k}}=\sqrt{m^{2}+c^{2} k^{2}}$, see the Appendix for details. Even though the Hamiltonian Eq. (2) depends on $\mu_{r}$, its ground state is, in fact, independent of $\mu_{r}$. This is a manifestation of the silver blaze problem, i.e., at zero temperature, thermodynamical observables are independent of the chemical potential up to some critical value [31], namely, $\mu_{r}^{2} \leqslant m^{2}$. Specifically, as shown in the Appendix, we find a factorized ground-state wave-functional $\Psi_{0}\left[\phi_{1}, \phi_{2}\right]=$ $\Psi_{\Omega_{\mathbf{k}}}\left[\phi_{1}\right] \Psi_{\Omega_{\mathbf{k}}}\left[\phi_{2}\right]$ with

$$
\Psi_{\Omega_{\mathbf{k}}}[\phi] \propto \exp \left\{-\frac{1}{2} \int d^{D} \mathbf{k} K \Omega_{\mathbf{k}} \phi^{2}\right\} .
$$

This expression explicitly shows that quantum fluctuations in the ground state are only sensitive to the combined excitation energy $\omega_{\mathbf{k}}^{+}+\omega_{\mathbf{k}}^{-}=2 \Omega_{\mathbf{k}}$ as a consequence of the conservation of the charge associated with the $O(2)$ invariance of the full theory. Furthermore, it shows that entanglement entropy $S$ is the sum of two contributions, stemming from the uncoupled Gaussian fluctuations of the $\phi_{1}$ and $\phi_{2}$ fields. Entanglement entropy, therefore, obeys an area law containing a nonuniversal UV-cutoff-dependent part $a_{0}$ and a universal contribution $a_{1}$ governed by the correlation length $\xi=c / m[13,32]$, see also the Appendix,

$$
S / \mathcal{A}=a_{0}-2 a_{1}(\xi),
$$

where $a_{1}(\xi)=1 /(12 \xi) \quad$ in $\quad D=2 \quad$ and $\quad a_{1}(\xi)=$ $\left(24 \pi \xi^{2}\right)^{-1} \ln \xi$ in $D=3$.

\section{ORDERED PHASE}

In this part $\left(\mu_{r}^{2}>m^{2}\right)$, we focus on the ordered phase for systems in $D=2,3$ spatial dimensions as in $D=1$ longrange order is not stable, and the physics is not captured by the Gaussian approximation we consider. Here, $\phi_{1}$ and $\phi_{2}$, respectively, capture amplitude and phase fluctuations of the order parameter $\psi^{(0)}=\sqrt{\left(\mu_{r}^{2}-m^{2}\right) / c_{4}}$, and we find

$$
\begin{aligned}
\mathcal{H}_{\text {ord. }}^{(2)}(\mathbf{k})= & \frac{1}{2 K}\left(\pi_{1}^{2}+\pi_{2}^{2}\right)+\mu_{r}\left(\phi_{1} \pi_{2}-\phi_{2} \pi_{1}\right) \\
& +\frac{K}{2}\left[\phi_{1}^{2}\left(c^{2} k^{2}+3 \mu_{r}^{2}-2 m^{2}\right)+\phi_{2}^{2}\left(c^{2} k^{2}+\mu_{r}^{2}\right)\right] .
\end{aligned}
$$

We first discuss the relativistic case $\left(\mu_{r}=0, m^{2}<0\right)$. In this situation, amplitude and phase fluctuations are decoupled. They are the normal modes of the theory, namely, the gapped Higgs mode with frequency $\omega_{\mathbf{k}}^{\mathrm{H}}=\sqrt{c^{2} k^{2}-2 m^{2}}$ (the Higgs gap is $\Delta_{\mathrm{H}}=|m| \sqrt{2}$ ) and the gapless Goldstone mode with $\omega_{\mathbf{k}}^{\mathrm{G}}=c k$. As a consequence, the ground-state wave-functional factorizes: $\Psi_{0}\left[\phi_{1}, \phi_{2}\right]=\Psi_{\omega_{\mathrm{k}}^{\mathrm{H}}}\left[\phi_{1}\right] \Psi_{\omega_{\mathrm{k}}^{\mathrm{G}}}\left[\phi_{2}\right]$ so that entanglement entropy is again the sum of two contributions: $S=S_{\mathrm{H}}+$ $S_{\mathrm{G}}$. Both contributions satisfy an area law in $D \geqslant 2$ spatial dimensions, and the area-law prefactor of $S_{\mathrm{G}}$ (stemming from the gapless Goldstone mode) is nonuniversal $[19,33-36] . S_{\mathrm{H}}$, on the other hand, contains a universal contribution $-a_{1}\left(\xi_{\mathrm{H}}\right)$ to the area-law prefactor [13], governed by the Higgs correlation length $\xi_{\mathrm{H}}=c / \Delta_{\mathrm{H}}$. The expression of $a_{1}(\xi)$ is given after Eq. (4). This prediction is one of our main results. It shows that, upon crossing the $O(2)$ quantum-critical point, entanglement entropy displays a universal singularity, directly stemming from the Higgs mode going gapless at the critical point. Such a dependence on the correlation length was known in the (gapped) disordered phase [32] but not in the (gapless) 


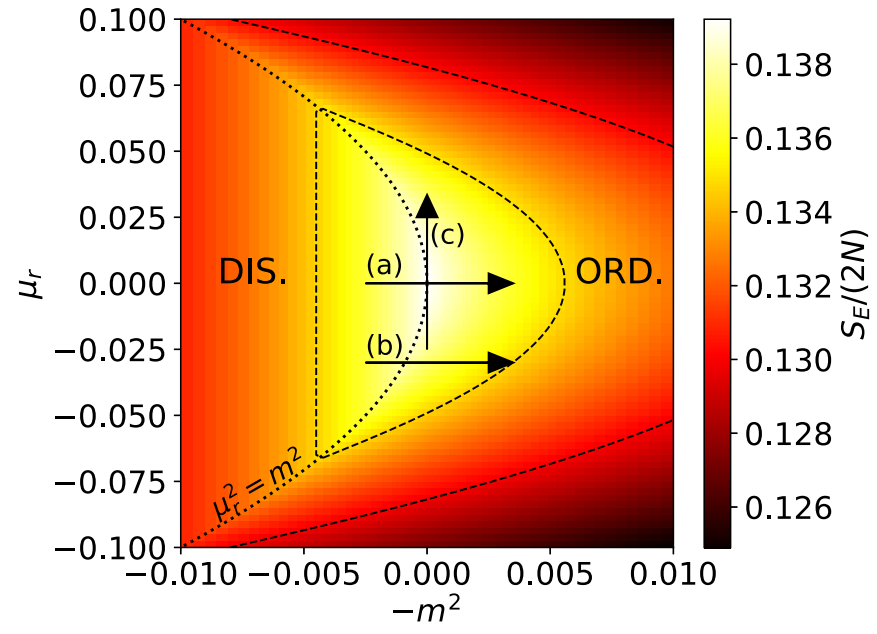

FIG. 1. Entanglement entropy per unit area across the twodimensional (2D) phase diagram of the model Eq. (1). Region $A$ is half of a $N \times N$ torus $(N=100)$. The dotted line denotes the critical line $\mu_{r}^{2}=m^{2}$ separating the disordered (DIS.) and the ordered phase (ORD.). The dashed lines correspond to constant entanglement entropy. The arrows mark trajectories where the area-law prefactor is plotted in Fig. 2.

ordered phase where it is a consequence of the Higgs gap. This singularity has been observed in previous numerical studies [37-39], and our paper provides its analytical explanation at a field-theory level.

In the general nonrelativistic case $\left(\mu_{r} \neq 0\right)$, the normal modes (a gapped $\phi_{\mathrm{H}}$ and a gapless $\phi_{\mathrm{G}}$ mode) are linear combinations of $\phi_{1}$ and $\phi_{2}$, see the Appendix for details. The Goldstone mode maintains a linear dispersion at low-energy $\omega_{\mathbf{k}}^{\mathrm{G}} \approx c_{\mathrm{G}} k$, albeit with a modified velocity $c_{\mathrm{G}}=c \sqrt{\left(\mu_{r}^{2}-m^{2}\right) /\left(3 \mu_{r}^{2}-m^{2}\right)}$. The Higgs gap is $\Delta_{\mathrm{H}}=$ $\sqrt{6 \mu_{r}^{2}-2 m^{2}}$, and, most importantly, it remains finite at the phase transition $\left(\Delta_{H}=2\left|\mu_{r}\right|\right)$. In this nonrelativistic case, entanglement entropy cannot be separated into two additive contributions stemming from amplitude and phase fluctuations. We can, however, evaluate it numerically, see the Appendix, following well-established methods to compute entanglement entropy for Gaussian states [35,40]. In Fig. 1, we show entanglement entropy in the $\left(-m^{2}, \mu_{r}\right)$ phase diagram for $D=2$. Interestingly, the nonrelativistic quantum critical points are actually not critical, as all correlation functions decay exponentially with a correlation length governed by the gap $\xi=c /\left(2\left|\mu_{r}\right|\right)$. This feature is clear from the discussion in the disordered phase: The ground state is independent of $\mu_{r}$ as long as $\mu_{r} \leqslant m$, including at the critical point $\mu_{r}=m$. Therefore, it is identical to the ground state with $\mu_{r}=0$ and $m>0$, namely, strictly inside the disordered phase. This observation is key to understand the low-energy properties of ferrimagnets as discussed at the end of the paper.

The results presented above are very general and affect a large variety of models whose low-energy dynamics is captured by the Lagrangian Eq. (1). In the following, we provide two prominent examples where our results can be directly applied.
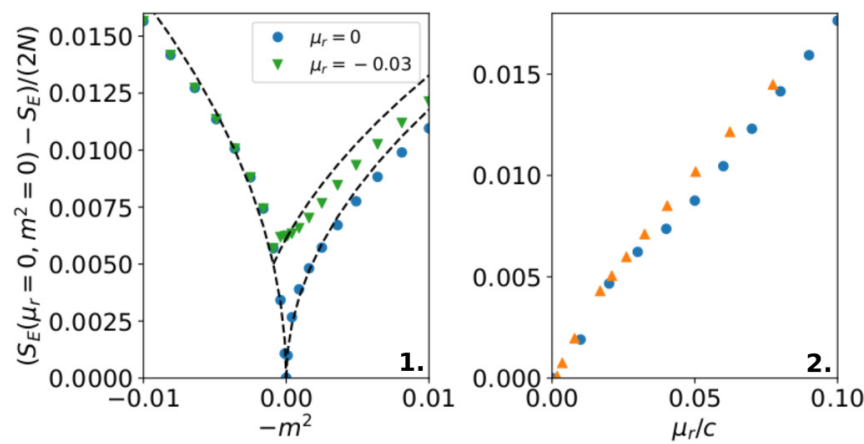

FIG. 2. Singular behavior of the area-law coefficient in the thermodynamic limit. (1) Trajectories (a) and (b) of Fig. 1. The dashed lines correspond to Eq. (4) using $\xi=c / m$ in the disordered phase and $\xi_{H}=c / \Delta_{H}$ in the ordered one. (2) Trajectory (c). Triangles: 2D Bose-Hubbard results (slave-boson method of Ref. [39]); circles: numerics on the Gaussian field theory explained in the Appendix.

\section{THE BOSE-HUBBARD MODEL}

We first consider a paradigmatic instance of the $O(2)$ quantum phase transition: the MI to SF transition in the Bose-Hubbard (BH) model in $D=2$ dimensions [41]. The Hamiltonian describes a square-lattice Bose gas with contact interactions at zero temperature [42],

$$
H=-J \sum_{\langle i, j\rangle}\left(b_{i}^{\dagger} b_{j}+\text { H.c. }\right)+\frac{U}{2} \sum_{i} b_{i}^{\dagger} b_{i}^{\dagger} b_{i} b_{i}-\mu \sum_{i} b_{i}^{\dagger} b_{i},
$$

where $b_{i}\left(b_{i}^{\dagger}\right)$ are bosonic annihilation (creation) operators on site $i=1, \ldots, N, \mu$ is the chemical potential, $J$ is the hopping amplitude, and $U$ is proportional to the two-bosons interaction strength. Near the critical point, an effective lowenergy description of the system applies $[1,42,43]$. This effective description coincides with the Lagrangian Eq. (1) where the order parameter is proportional to the vacuum expectation value of the bosonic annihilation operator $\psi(\vec{r}, t) \propto\left\langle b_{i}(t)\right\rangle$. The coefficients of the Lagrangian Eq. (1) can be expressed in terms of the Bose-Hubbard parameters [1,44], see the Appendix.

The transition between the MI and the SF may occur via two different mechanisms: either the interaction strength is varied at the fixed integer filling fraction or the particle number is changed by adjusting the chemical potential. In the former case, the low-energy description becomes relativistic [22] so that $\mu_{r}=0$, see the Appendix. The phase transition occurs across the $O(2)$ quantum-critical point where the Higgs correlation length $\xi_{\mathrm{H}}$ diverges. In the latter case, relativistic invariance is not present $\left(\mu_{r} \neq 0\right)$, and the Higgs gap remains finite at the transition. The comparison between our results for the entanglement entropy along these two paths in the phase diagram and the analytical formula Eq. (4) using $\xi=$ $c / m$ in the disordered phase and $\xi_{\mathrm{H}}=c / \Delta_{\mathrm{H}}$ in the ordered one is shown in Fig. 2 (panel 1). The agreement is, in all cases, extremely good. Finally, on the SF side, touching the $O(2)$ point by varying $\mu$ at fixed $U=U_{c}$, we predict that entanglement entropy behaves linearly with $\mu$, in agreement with the numerical results of Ref. [39] [Fig. 2 (panel 2)]. 


\section{NONRELATIVISTIC NAMBU-GOLDSTONE BOSONS}

The Lagrangian Eq. (1) with $c_{4}=0$ and $\mu_{r}^{2}=m^{2}$ has been proposed as the low-energy description of $\mathrm{NG}$ bosons without Lorentz invariance [6,7] in a physical situation where the system has a rotational $O(3)$ symmetry which is broken down to $O(2)$, i.e., the ground state chooses a particular orientation. Following the general classification given in Refs. [45-47], the original complex scalar field can be identified with two NG fields $\psi(\vec{r}, t)=\pi_{1}(\vec{r}, t)+i \pi_{2}(\vec{r}, t)$. One of them corresponds to a type-B NG boson and has a quadratic dispersion relation [7]; the other one is a so-called gapped partner [27-30].

From our previous discussion, one sees that these systems are at the critical point between the disordered and the ordered phase at finite chemical potential $\mu_{r}=m$. They exhibit a finite correlation length $\xi=c / m$, although they present quadratic gapless excitations. Therefore, entanglement entropy should obey an area law [see Eq. (4)]. This should be compared with the relativistic case where (type-A) NG bosons acquire a linear dispersion relation and a diverging correlation length.

Here, we focus on the $D=1$ case where entanglement entropy is expected to scale according to $S_{E} \sim \ln \xi[12,13,51]$. We validate our approach by considering a particular family of systems, namely, ferrimagnets, which are spin systems living on two sublattices $A_{1} \cup A_{2}$. On the $A_{1}$ sublattice, we define $\vec{S}_{i}$ as spin- $s_{1}$ operators, and on the $A_{2}$ sublattice, we define $\vec{\tau}_{j}$ as spin- $s_{2}$ operators. Spins interact via Heisenberg-type exchange interactions with Hamiltonian $H_{\text {ferrimag. }}=J \sum_{\langle i, j\rangle} \vec{S}_{i}$. $\vec{\tau}_{j}$. Typically, the ground state of the system exhibits ferrimagnetic order, i.e., an antialignment of the spins living on different sublattices. In this case, the different magnitude of the spins induces a total magnetization density in the system $m=\left\langle S^{z}\right\rangle / N \sim\left|s_{1}-s_{2}\right|$. This is an indicator of the nonzero expectation value of the commutator of two broken charges $\left\langle\left[S^{x}, S^{y}\right]\right\rangle \neq 0$, which breaks Lorentz invariance [7].

The low-energy effective description of ferrimagnets is the Lagrangian Eq. (1) with $c_{4}=0$ and $\mu_{r}^{2}=m^{2}$ [52], whose low-energy excitations are $\omega_{\mathbf{k}}^{ \pm}=\sqrt{m^{2}+c^{2} k^{2}} \pm m$. Thus, we expect quadratic type-B NG bosons $\omega_{\mathbf{k}}^{-}=\rho k^{2}$ and a gapped partner $\omega_{\mathbf{k}}^{+}=\Delta$, where $\rho$ and $\Delta$ are the spin stiffness and energy gap, respectively. In terms of the coefficients of the Lagrangian Eq. (1), we identify $\rho=c^{2} /(2 m)$ and $\Delta=2 m$, which allows us to write the expression for the correlation length (in units of the lattice spacing),

$$
\xi=2 \sqrt{\frac{\rho}{\Delta}} .
$$

We compare the prediction Eq. (7) for the correlation length with various numerical computations on $H_{\text {ferrimag. }}$ where a very short correlation length was found together with gapless excitations [48,50,53], two key features which are clearly present at the field-theory level. Indeed, Eq. (7) allows us to predict the value of the correlation length for several ferrimagnets using previously obtained numerical results for $\rho$ and $\Delta$. The results are summarized in Fig. 3. Our prediction for $\xi$ is systematically smaller than those predicted by spinwave theory $\xi^{-1}=\ln \left(s_{1} / s_{2}\right)$ [48] and closer to the (more accurate) value obtained using matrix product states $[48,50]$ for $\left(s_{1}=1, s_{2}=1 / 2\right)$. In addition to existing results in the literature, we also carried out iDMRG simulations [54] and
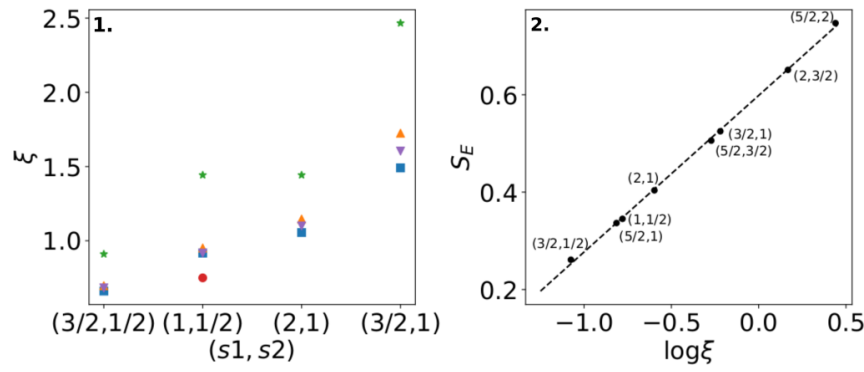

FIG. 3. Panel 1: Correlation lengths for different ferrimagnetic systems with spins $\left(s_{1}, s_{2}\right)$. Predicted values from Eq. (7) using previously computed values for $\rho$ and $\Delta$ from the spin wave [48] (stars), interacting spin wave [48] (upper triangles), and Monte Carlo and exact diagonalizations [48,49] (squares). For $\left(s_{1}, s_{2}\right)=$ $(1,1 / 2)$, we include the value obtained using matrix product states in Refs. [48,50] (circles). Lower triangles are results obtained using infinite density-matrix renormalization group (iDMRG). Panel 2: Entanglement entropy for different ferrimagnets as a function of the extracted correlation length.

computed the correlation lengths for different spin values $s_{1}$ and $s_{2}$. We found a good agreement with the prediction Eq. (7) using existing Monte Carlo data for the values of $\rho$ and $\Delta$. Finally, we observe that these values of $\xi$ are also consistent with entanglement entropy scaling as $S \sim \ln \xi$.

\section{OUTLOOK}

We have investigated the area-law prefactor of entanglement entropy in nonrelativistic low-energy field theories with $O(2)$ symmetry. Our predictions have been successfully confronted with two prominent examples from condensedmatter physics: the Mott insulator to superfluid transition and nonrelativistic Nambu-Goldstone bosons in ferrimagnets. Our findings, which could be tested in quantum simulators $[55,56]$, also apply to particle-physics models with a nonzero chemical potential.

\section{ACKNOWLEDGMENTS}

The authors thank J. Martorell for a careful reading of the paper. The authors also thank T. Fiol, L. Tagliacozzo, J. Taron, and G. Sierra for useful comments and discussions. This work was partially funded by MINECO (Spain) (Grant No. FIS2017-87534-P and Severo Ochoa Grant No. SEV-2015-0522), the European Union Regional Development Fund within the ERDF Operational Program of Catalunya (Project QUASICAT/QuantumCat), the Fundacio Mir-Puig and Cellex through an ICFO-MPQ postdoctoral fellowship, and the Generalitat de Catalunya (Grant No. SGR 1381 and the CERCA Programme).

\section{APPENDIX: DETAILS OF THE DERIVATIONS}

In this Appendix, we provide further details about:

(1) The representation of the ground-state wave functional in real space in the disordered phase and its connection with entanglement entropy. 
(2) The Gaussian approximation in the ordered phase and the computation of entanglement entropy.

(3) The explicit relation with the parameters of the BoseHubbard model.

\section{Disordered phase}

We consider the free nonrelativistic theory describing the dynamics of a complex scalar field $\psi=\left(\phi_{1}+i \phi_{2}\right) / \sqrt{2}$, whose Lagrangian density in $(D+1)$-dimensional spacetime is given by

$$
\mathcal{L} / K=\left|\left(\partial_{t}-i \mu_{r}\right) \psi\right|^{2}-c^{2}|\nabla \psi|^{2}-m^{2}|\psi|^{2}-c_{4}|\psi|^{4} .
$$

The theory is nonrelativistic because of the presence of the term $\mu_{r} \neq 0$. We work with the Hamiltonian formulation of the theory. We introduce the canonical moments $\pi_{1 / 2}=\frac{\delta \mathcal{L}}{\delta\left(\partial_{t} \phi_{1 / 2}\right)}=K\left(\partial_{t} \phi_{1 / 2} \pm \mu_{r} \phi_{2 / 1}\right)$. The Hamiltonian operator reads in Fourier space,

$$
\begin{aligned}
\mathcal{H}(\mathbf{k})= & \frac{1}{2 K}\left(\pi_{1}^{2}+\pi_{2}^{2}\right)+\frac{K}{2}\left(m^{2}+c^{2} k^{2}\right)\left(\phi_{1}^{2}+\phi_{2}^{2}\right) \\
& +\mu_{r}\left(\phi_{1} \pi_{2}-\phi_{2} \pi_{1}\right),
\end{aligned}
$$

where $\left[\phi_{a}(\mathbf{k}), \pi_{b}\left(\mathbf{k}^{\prime}\right)\right]=i \delta_{a, b} \delta\left(\mathbf{k}-\mathbf{k}^{\prime}\right)$ and $\left[\phi_{a}(\mathbf{k}), \phi_{b}\left(\mathbf{k}^{\prime}\right)\right]=$ $\left[\pi_{a}(\mathbf{k}), \pi_{b}\left(\mathbf{k}^{\prime}\right)\right]=0$.

We are now neglecting the interactions, setting $c_{4}=0$. Therefore, the results derived in this section can be seen as performing a Gaussian approximation in the disordered phase described in the main text. We look for a canonical transformation of the form

$$
\begin{aligned}
& \pi_{ \pm}=\frac{1}{\sqrt{2}}\left[\sqrt{\frac{\Gamma_{ \pm}}{K \Omega}} \pi_{1} \pm \sqrt{K \Omega \Gamma_{ \pm}} \phi_{2}\right], \\
& \phi_{ \pm}=\frac{1}{\sqrt{2}}\left[\sqrt{\frac{K \Omega}{\Gamma_{ \pm}}} \phi_{1} \mp \frac{1}{\sqrt{K \Omega \Gamma_{ \pm}}} \pi_{2}\right] .
\end{aligned}
$$

One can verify that choosing $\Gamma_{ \pm}=\sqrt{m^{2}+c^{2} k^{2}} \mp \mu_{r}$ and $\Omega=\sqrt{m^{2}+c^{2} k^{2}}$, the Hamiltonian reads

$$
\mathcal{H}(\mathbf{k})=\frac{1}{2}\left(\pi_{+}^{2}+\pi_{-}^{2}\right)+\frac{1}{2}\left(\Gamma_{+}^{2} \phi_{+}^{2}+\Gamma_{-}^{2} \phi_{-}^{2}\right) .
$$

Finally the Hamiltonian Eq. (A4) can be diagonalized by introducing a set of normal modes $( \pm)$ defined by the annihilation operators,

$$
a_{ \pm}=\sqrt{\frac{\Gamma_{ \pm}}{2}} \phi_{ \pm}+\frac{i}{\sqrt{2 \Gamma_{ \pm}}} \pi_{ \pm},
$$

which allows one to write the Hamiltonian in an harmonicoscillator form

$$
\mathcal{H}(\mathbf{k})=\Gamma_{+}\left(a_{+}^{\dagger} a_{+}+\frac{1}{2}\right)+\Gamma_{-}\left(a_{-}^{\dagger} a_{-}+\frac{1}{2}\right) .
$$

Because of this harmonic-oscillator form, we know that the ground-state $\left|\Psi_{0}\right\rangle$ is uniquely determined by the condition $a_{+}(\mathbf{k})\left|\Psi_{0}\right\rangle=a_{-}(\mathbf{k})\left|\Psi_{0}\right\rangle=0, \forall(\mathbf{k})$. In the Schrödinger representation, the ground-state wave functional is the Gaussian,

$\Psi_{0}\left[\phi_{+}, \phi_{-}\right]=\mathcal{N} \exp \left(-\frac{1}{2} \int d^{D} \mathbf{k}\left[\phi_{+} \Gamma_{+} \phi_{+}+\phi_{-} \Gamma_{-} \phi_{-}\right]\right)$, where $\mathcal{N}$ is a normalization factor. Turning back to the original $\phi_{1}$ and $\phi_{2}$ fields using the canonical transformation Eq. (A4), one obtains

$\Psi_{0}\left[\phi_{1}, \pi_{2}\right]=\mathcal{N} \exp \left(-\frac{1}{2} \int d^{D} \mathbf{k}\left[\phi_{1} K \Omega \phi_{1}+\pi_{2} \frac{1}{K \Omega} \pi_{2}\right]\right)$,

or equivalently

$\Psi_{0}\left[\phi_{1}, \phi_{2}\right]=\mathcal{N} \exp \left(-\frac{1}{2} \int d^{D} \mathbf{k}\left[\phi_{1} K \Omega \phi_{1}+\phi_{2} K \Omega \phi_{2}\right]\right)$,

which coincides with the ground-state wave functional of two relativistic scalar fields of kinetic mass $K$ and dispersion relation $\Omega(\mathbf{k})=\sqrt{m^{2}+c^{2} k^{2}}$. This result explicitly shows that the ground state is independent of $\mu_{r}$ as long as we stay in the disordered phase (namely, as long as $\mu_{r}^{2} \leqslant m^{2}$ ). From this ground-state wave functional, one can readily see that the two-point function $\left\langle 0\left|\psi(\vec{x}, t) \psi^{\dagger}(\vec{y}, t)\right| 0\right\rangle$ has an exponential decay $\exp \{-r / \xi\}$ for long-distance $r=|\vec{x}-\vec{y}|$ given by the correlation length $\xi=c / m$. Since we have been able to express the ground-state wave functional as the one of a relativistic complex scalar field with a mass $m$, the computation of entanglement entropy will be based on the known results obtained for the relativistic case [10-14].

Consider that we perform a spatial partition of the system into two parts $\mathcal{A}$ and $\mathcal{B}$. The entanglement entropy is defined as $S_{E}=-\operatorname{Tr}_{\mathcal{A}}\left\{\rho_{\mathcal{A}} \ln \rho_{\mathcal{A}}\right\}$, where $\rho_{\mathcal{A}}=\operatorname{Tr}_{\mathcal{B}} \rho$ is the reduced density matrix obtained by tracing out the degrees of freedom living on $\mathcal{B}$ and $\rho=|0\rangle\langle 0|$ is the density matrix which can be represented using the ground-state wave-functional Eq. (A7). If the system presents a finite correlation length $\xi$, the leading area-law term of entanglement entropy is given by $[13,51]$

$$
S_{E}=-A \frac{N}{12} \int \frac{d^{D-1} k_{\perp}}{(2 \pi)^{D-1}} \ln \frac{k_{\perp}^{2}+\xi^{-2}}{k_{\perp}^{2}+a^{-2}},
$$

where $\epsilon=1 / a$ is the UV cutoff, $A$ is the area of the boundary separating the two parts of the system $\mathcal{A}$ and $\mathcal{B}$, and $N=2$ is the number of free scalar fields. One can see that this integral contains a term which is UV-cutoff dependent and that makes it nonuniversal. On the other hand, there is a finite universal term that goes, such as $\xi^{1-D}$ which can be explicitly manifested by taking the derivative $\partial S_{E} / \partial \xi^{-2}$,

$$
\frac{\partial S_{E}}{\partial \xi^{-2}}=-A \frac{N}{12} \int \frac{d^{D-1} k_{\perp}}{(2 \pi)^{D-1}} \frac{1}{k_{\perp}^{2}+\xi^{-2}},
$$

which leads to the well-known result for $D=2[13,51]$,

$$
S_{E} / A=-\frac{1}{6 \xi}+a_{0},
$$

where $a_{0}$ stands for some nonuniversal divergent constant cutoff dependent. In this way, one can compute $\Delta S_{E}=S_{E}(\xi \rightarrow$ $\infty)-S_{E}(\xi)$ which should not depend on the UV cutoff, and therefore, it will be universal in this sense. 


\section{Normal modes in the ordered phase}

We consider the Lagrangian density,

$\mathcal{L} / K=\left|\left(\partial_{t}-i \mu_{r}\right) \psi\right|^{2}-c^{2}|\nabla \psi|^{2}-m^{2}|\psi|^{2}-c_{4}|\psi|^{4}$.

Introducing $\quad \psi=\left(\phi_{1}+i \phi_{2}\right) / \sqrt{2}, \quad$ and $\quad \pi_{1 / 2}=\frac{\partial \mathcal{L}}{\partial\left(\partial_{t} \phi_{1 / 2}\right)}=$ $K\left(\partial_{t} \phi_{1 / 2} \pm \mu_{r} \phi_{2 / 1}\right)$, we have the equivalent Hamiltonian density,

$$
\begin{aligned}
\mathcal{H}= & \pi_{1} \partial_{t} \phi_{1}+\pi_{2} \partial_{t} \phi_{2}-\mathcal{L} \\
= & \frac{1}{2 K}\left(\pi_{1}^{2}+\pi_{2}^{2}\right)+\mu_{r}\left(\pi_{2} \phi_{1}-\pi_{1} \phi_{2}\right)+\frac{K m^{2}}{2}\left(\phi_{1}^{2}+\phi_{2}^{2}\right) \\
& +\frac{K c^{2}}{2}\left[\left(\nabla \phi_{1}\right)^{2}+\left(\nabla \phi_{2}\right)^{2}\right]+\frac{K c_{4}}{4}\left(\phi_{1}^{2}+\phi_{2}^{2}\right)^{2} .
\end{aligned}
$$

We focus on the ordered phase, namely, $m^{2}<\mu_{r}^{2}$. Imposing that $\delta \mathcal{H}=0$, we obtain the mean-field solution $\phi_{2}^{(0)}=0, \pi_{1}^{(0)}=0, \pi_{2}^{(0)}=-K \mu_{r} \phi_{1}^{(0)}$, and $\phi_{1}^{(0)}=$ $\pm \sqrt{\left(\mu_{r}^{2}-m^{2}\right) / c_{4}}$. Expanding around this mean-field solution (namely, $x \rightarrow x^{(0)}+x$ for $x=\pi_{1 / 2}, \phi_{1 / 2}$ ), and neglecting terms of order $\mathcal{O}\left(x^{3}\right)$, we obtain

$$
\begin{aligned}
\mathcal{H}^{(2)}= & \frac{1}{2 K}\left(\pi_{1}^{2}+\pi_{2}^{2}\right)+\mu_{r}\left(\pi_{2} \phi_{1}-\pi_{1} \phi_{2}\right)+\frac{K}{2} \mu_{r}^{2}\left(\phi_{1}^{2}+\phi_{2}^{2}\right) \\
& +K\left(\mu_{r}^{2}-m^{2}\right) \phi_{1}^{2}+\frac{K c^{2}}{2}\left[\left(\nabla \phi_{1}\right)^{2}+\left(\nabla \phi_{2}\right)^{2}\right] . \quad(\mathrm{A} 15)
\end{aligned}
$$

Clearly, when $\mu_{r}=0, \phi_{1}$, and $\phi_{2}$ are decoupled and represent the normal modes (namely, the Higgs and Goldstone modes of the ordered phase). They contribute independently to entanglement entropy of a subsystem, which, therefore, reads $S=S_{\text {Goldstone }}+S_{\text {Higgs }}$. However, for $\mu_{r} \neq 0$ they are coupled.

\section{a. Diagonalization of the Hamiltonian}

Going to Fourier space, the Hamiltonian density reads

$$
\mathcal{H}^{(2)}(k)=\frac{1}{2}\left(\begin{array}{llll}
\phi_{1} & \phi_{2} & \pi_{1} & \pi_{2}
\end{array}\right) H(k)\left(\begin{array}{l}
\phi_{1} \\
\phi_{2} \\
\pi_{1} \\
\pi_{2}
\end{array}\right),
$$

where $H$ is of the form

$$
H(k)=\left(\begin{array}{cccc}
A_{1} & 0 & 0 & C \\
0 & A_{2} & -C^{\prime} & 0 \\
0 & -C^{\prime} & B_{1} & 0 \\
C & 0 & 0 & B_{2}
\end{array}\right),
$$

with $A_{1}=K\left(3 \mu_{r}^{2}-2 m^{2}+c^{2} k^{2}\right), A_{2}=K\left(\mu_{r}^{2}+c^{2} k^{2}\right), B_{1}=$ $B_{2}=1 / K, C=C^{\prime}=\mu_{r}$. In order to diagonalize the Hamiltonian, we look for a canonical transformation

$$
\left(\begin{array}{l}
\phi_{1} \\
\phi_{2} \\
\pi_{1} \\
\pi_{2}
\end{array}\right)=W\left(\begin{array}{l}
q_{1} \\
q_{2} \\
p_{1} \\
p_{2}
\end{array}\right)
$$

where $W$ is chosen of the form

$$
W=\left(\begin{array}{cccc}
\alpha_{1} & \alpha_{2} & 0 & 0 \\
0 & 0 & \beta_{1} & \beta_{2} \\
0 & 0 & \gamma_{1} & \gamma_{2} \\
\delta_{1} & \delta_{2} & 0 & 0
\end{array}\right)
$$

$W$ represents a canonical transformation iff $W J W^{T}=J$ with

$$
J=\left(\begin{array}{rrrr}
0 & 0 & 1 & 0 \\
0 & 0 & 0 & 1 \\
-1 & 0 & 0 & 0 \\
0 & -1 & 0 & 0
\end{array}\right)
$$

Introducing the matrices $X=\left(\begin{array}{ll}\alpha_{1} & \alpha_{2} \\ \delta_{1} & \delta_{2}\end{array}\right)$ and $Y=\left(\begin{array}{cc}\gamma_{1} & \gamma_{2} \\ -\beta_{1} & -\beta_{2}\end{array}\right)$, $W$ is a canonical transformation iff $X Y^{T}=$ Id. The aim of the canonical transformation $W$ is to bring the matrix $H$ into the form

$$
W^{T} H W=\left(\begin{array}{cccc}
\omega_{1}^{2} & 0 & 0 & 0 \\
0 & \omega_{2}^{2} & 0 & 0 \\
0 & 0 & 1 & 0 \\
0 & 0 & 0 & 1
\end{array}\right) .
$$

This is equivalent to having $X^{T} M_{1} X=\left(\begin{array}{cc}\omega_{1}^{2} & 0 \\ 0 & \omega_{2}^{2}\end{array}\right)$ with $M_{1}=$ $\left(\begin{array}{cc}A_{1} & C \\ C & B_{2}\end{array}\right)$ and $Y^{T} M_{2} Y=\mathrm{Id}$ with $M_{2}=\left(\begin{array}{cc}B_{1} & C^{\prime} \\ C^{\prime} & A_{2}\end{array}\right)$. The diagonalization may be achieved by noting that $\left(\begin{array}{cc}\omega_{1}^{2} & 0 \\ 0 & \omega_{2}^{2}\end{array}\right)=$ $X^{T} M_{1} X Y^{T} M_{2} Y=X^{T} M_{1} M_{2} Y$ where we use the fact that $X Y^{T}=$ Id. Since $X^{T}=Y^{-1}$, we can now diagonalize $M_{1} M_{2}$ to obtain $X, Y, \omega_{1}$, and $\omega_{2}$. This is performed in two steps. First, $M_{1} M_{2}$ is diagonalized, yielding $\tilde{X}^{T} M_{1} M_{2} \tilde{Y}=$ $\operatorname{diag}\left(\omega_{1}^{2}, \omega_{2}^{2}\right) . Y$ is then obtained by properly normalizing the columns of $\tilde{Y}$. We initially have $\tilde{Y}^{T} M_{2} \tilde{Y}=\operatorname{diag}\left(\lambda_{1}^{2}, \lambda_{2}^{2}\right)$. Finally, we obtain the normal modes by choosing $Y=$ $\tilde{Y} \operatorname{diag}\left(1 / \lambda_{1}, 1 / \lambda_{2}\right)$ and $X^{T}=Y^{-1}$. Then, the Hamiltonian reads

$$
\mathcal{H}^{(2)}(k)=\sum_{i=1}^{2} \frac{1}{2}\left[p_{i}(k) p_{i}(-k)+\omega_{i}(k)^{2} q_{i}(k) q_{i}(-k)\right],
$$

where $\left(\begin{array}{c}\phi_{1} \\ \pi_{2}\end{array}\right)=X\left(\begin{array}{c}q_{1} \\ q_{2}\end{array}\right)$ and $\left(\begin{array}{c}\pi_{1} \\ -\phi_{2}\end{array}\right)=Y\left(\begin{array}{c}p_{1} \\ p_{2}\end{array}\right)$.

\section{b. Eigenmodes}

The eigenfrequencies are as follows:

$$
\omega_{ \pm}^{2}=c^{2} k^{2}+3 \mu_{r}^{2}-m^{2} \pm \sqrt{4 \mu_{r}^{2} c^{2} k^{2}+\left(3 \mu_{r}^{2}-m^{2}\right)^{2}} .
$$

For the gapless Goldstone mode, we find, expanding at low $k$,

$$
\omega_{\mathrm{G}}^{2}=\omega_{-}^{2}=c^{2} k^{2} \frac{\mu_{r}^{2}-m^{2}}{3 \mu_{r}^{2}-m^{2}}+\mathcal{O}\left(k^{4}\right),
$$

Hence, a modified sound velocity $c_{\mathrm{G}}=c \sqrt{\frac{\mu_{r}^{2}-m^{2}}{3 \mu_{r}^{2}-m^{2}}}$ such that $\omega_{\mathrm{G}}=c_{\mathrm{G}} k+\mathcal{O}\left(k^{2}\right)$. For the gapped Higgs mode, we find, instead,

$$
\omega_{\mathrm{H}}^{2}=\omega_{+}^{2}=6 \mu_{r}^{2}-2 m^{2}+c^{2} k^{2} \frac{5 \mu_{r}^{2}-m^{2}}{3 \mu_{r}^{2}-m^{2}}+\mathcal{O}\left(k^{4}\right) .
$$

This defines the Higgs gap: $\Delta_{\mathrm{H}}=\sqrt{6 \mu_{r}^{2}-2 m^{2}}$ and the Higgs velocity: $c_{\mathrm{H}}=c \sqrt{\frac{5 \mu_{r}^{2}-m^{2}}{3 \mu_{r}^{2}-m^{2}}}$ such that $\omega_{\mathrm{H}}=\sqrt{\Delta_{\mathrm{H}}^{2}+c_{\mathrm{H}}^{2} k^{2}}+$ $\mathcal{O}\left(k^{4}\right)$. 


\section{c. Correlation functions}

Correlation functions in the ground state are obtained according to

$$
\begin{aligned}
\left\langle\left(\begin{array}{l}
\phi_{1} \\
\pi_{2}
\end{array}\right)\left(\begin{array}{ll}
\phi_{1} & \pi_{2}
\end{array}\right)\right\rangle & =X\left(\begin{array}{cc}
\frac{1}{2 \omega_{1}} & 0 \\
0 & \frac{1}{2 \omega_{2}}
\end{array}\right) X^{T}, \\
\left\langle\left(\begin{array}{c}
\pi_{1} \\
-\phi_{2}
\end{array}\right)\left(\begin{array}{ll}
\pi_{1} & -\phi_{2}
\end{array}\right)\right\rangle & =Y\left(\begin{array}{cc}
\omega_{1} / 2 & 0 \\
0 & \omega_{2} / 2
\end{array}\right) Y^{T} \\
& =\frac{1}{4}\left\langle\left(\begin{array}{c}
\phi_{1} \\
\pi_{2}
\end{array}\right)\left(\begin{array}{ll}
\phi_{1} & \pi_{2}
\end{array}\right)\right\rangle^{-1},
\end{aligned}
$$

and $\left\langle\phi_{1} \phi_{2}\right\rangle=0=\left\langle\pi_{1} \pi_{2}\right\rangle,\left\langle\phi_{1} \pi_{1}\right\rangle=i / 2=\left\langle\phi_{2} \pi_{2}\right\rangle$.

\section{d. Entanglement entropy}

In order to compute the entanglement entropy of a subsystem $A$, one first forms the correlation matrix for the field degrees of freedom belonging to that subsystem: $\left\langle\phi_{1}(x) \phi_{2}\left(x^{\prime}\right)\right\rangle$ for $x, x^{\prime} \in A$, etc. We assume that $A$ contains $N$ sites. The correlation matrix is as follows:

$$
\mathcal{C}=\left(\begin{array}{cccc}
\left\langle\phi_{1} \phi_{1}\right\rangle & 0 & (i / 2) \mathrm{Id} & \left\langle\phi_{1} \pi_{2}\right\rangle \\
0 & \left\langle\phi_{2} \phi_{2}\right\rangle & \left\langle\phi_{2} \pi_{1}\right\rangle & (i / 2) \mathrm{Id} \\
(-i / 2) \mathrm{Id} & \left\langle\pi_{1} \phi_{2}\right\rangle & \left\langle\pi_{1} \pi_{1}\right\rangle & 0 \\
\left\langle\pi_{2} \phi_{1}\right\rangle & (-i / 2) \mathrm{Id} & 0 & \left\langle\pi_{2} \pi_{2}\right\rangle
\end{array}\right),
$$

where $\left\langle\phi_{1} \phi_{1}\right\rangle$ represents the $N \times N$ matrix $\left\langle\phi_{1}(x) \phi_{2}\left(x^{\prime}\right)\right\rangle$ for $x, x^{\prime} \in A$, and similarly for $\left\langle\phi_{1} \pi_{2}\right\rangle$, etc. Similar to the diagonalization of the Hamiltonian, the canonical transformation to the normal modes may be chosen of the form

$$
W=\left(\begin{array}{cccc}
\alpha_{1} & \alpha_{2} & 0 & 0 \\
0 & 0 & \beta_{1} & \beta_{2} \\
0 & 0 & \gamma_{1} & \gamma_{2} \\
\delta_{1} & \delta_{2} & 0 & 0
\end{array}\right)
$$

where now $\alpha_{i}, \beta_{i}, \gamma_{i}$, and $\delta_{i}$ are $N \times N$ matrices. The diagonalization of $\mathcal{C}$ goes along the same line as that of the Hamiltonian except for the fact that each symbol now represents a $N \times N$ matrix. Indeed, introducing $X=\left(\begin{array}{ll}\alpha_{1} & \alpha_{2} \\ \delta_{1} & \delta_{2}\end{array}\right)$ and $Y=\left(\begin{array}{cc}\gamma_{1} & \gamma_{2} \\ -\beta_{1} & -\beta_{2}\end{array}\right)$ and denoting

$$
\mathcal{C}=\left(\begin{array}{cccc}
A_{1} & 0 & (i / 2) \mathrm{Id} & C \\
0 & A_{2} & -C^{\prime} & (i / 2) \mathrm{Id} \\
-(i / 2) \mathrm{Id} & -C^{\prime T} & B_{1} & 0 \\
C^{T} & -(i / 2) \mathrm{Id} & 0 & B_{2}
\end{array}\right),
$$

we have that $\mathcal{C}=\operatorname{diag}\left(X^{T} M_{1} X, Y^{T} M_{2} Y\right)$ with $M_{1}=\left(\begin{array}{cc}A_{1} & C \\ C^{T} & B_{2}\end{array}\right)$ and $M_{2}=\left(\begin{array}{ll}B_{1} & C^{\prime T} \\ C^{\prime} & A_{2}\end{array}\right)$. Diagonalizing the matrix $M_{1} M_{2}$, one obtains eigenvalues of the form $\lambda_{i}^{2}=\left[\frac{1}{2}+\frac{1}{e^{\tilde{\omega}_{i}}-1}\right]^{2}$, where $\tilde{\omega}_{i}$ forms the (one-body) entanglement spectrum. The entanglement entropy of $A$ is finally obtained as

$S=\sum_{i}\left[\left(\lambda_{i}+\frac{1}{2}\right) \ln \left(\lambda_{i}+\frac{1}{2}\right)-\left(\lambda_{i}-\frac{1}{2}\right) \ln \left(\lambda_{i}-\frac{1}{2}\right)\right]$.
As a further simplification, we note that, if $A$ is periodic along a certain direction ( say $x$ ), the correlation matrix $\mathcal{C}$ is block diagonal with respect to the momentum $k_{x}$ along that direction. The diagonalization may, thus, be achieved separately in each momentum sector $k_{x}$, and the entanglement entropy is the sum of the contributions from the different sectors $k_{x}$.

\section{Relation with Bose-Hubbard parameters}

In this section, we write down explicitly the relations between the $\mathrm{BH}$ parameters and the effective parameters of our original model. We introduce the notations $K_{1} \equiv 2 \mu_{r} K, c_{2} \equiv$ $K\left(m^{2}-\mu_{r}^{2}\right)$, and $K_{3} \equiv K c^{2}$. For the first lobe $n=1$ and setting $U=1$, the following relations can be applied $[1,44]$

$$
\begin{aligned}
& c_{2}=\mu \frac{1-\mu}{1+\mu}-z J, \quad K_{1}=-\frac{\partial c_{2}}{\partial \mu}=\frac{\mu^{2}+2 \mu-1}{(1+\mu)^{2}}, \\
& K=-\frac{1}{2} \frac{\partial^{2} c_{2}}{\partial \mu^{2}}=\frac{2}{(1+\mu)^{3}}, \quad K_{3}=J .
\end{aligned}
$$

In these relations, the chemical potential of the $\mathrm{BH}$ model $\mu$ can be different from the chemical potential of the original model $\mu_{r}$ (we add the subscript $r$ to differentiate them). In order to simplify these relations, we expand around the tip of the lobe $z J_{c}=3-2 \sqrt{2}$ and $\mu_{c}=\sqrt{2}-1$, and we obtain the following relations:

$$
\begin{aligned}
c_{2} / K & \approx z \sqrt{2}\left(J_{c}-J\right)-\left(\mu-\mu_{c}\right)^{2}, \\
K_{1} & \approx 2 K\left(\mu_{c}\right)\left(\mu-\mu_{c}\right),
\end{aligned}
$$

where $K\left(\mu_{c}\right)=1 / \sqrt{2}$. If we compare these relations with the ones for the original models $c_{2} / K=m^{2}-\mu_{r}^{2}$ and $\mu_{r}=$ $K_{1} / 2 K$, we see that at leading order,

$$
m^{2}=z \sqrt{2}\left(J_{c}-J\right), \quad \mu_{r}=\mu-\mu_{c}, \quad c=\sqrt{\sqrt{2} J_{c}} .
$$

From Eq. (A12), we can see that inside the Mott-insulator phase $\Delta S_{E}$ will be independent of the chemical potential,

$$
\Delta S_{E} / A=\frac{1}{6} \sqrt{z} \sqrt{1-\frac{J}{J_{c}}}
$$

Inside the superfluid phase in the relativistic regime $\left(\mu=\mu_{c}\right)$, we obtain

$$
\Delta S_{E} / A=\frac{1}{12} \sqrt{2 z} \sqrt{\frac{J}{J_{c}}-1}
$$


[1] S. Sachdev, Quantum Phase Transitions, 2nd ed. (Cambridge University Press, Cambridge, UK, 2011).

[2] J. Goldstone, Il Nuovo Cimento 19, 154 (1961).

[3] J. Goldstone, A. Salam, and S. Weinberg, Phys. Rev. 127, 965 (1962).

[4] R. V. Lange, Phys. Rev. Lett. 14, 3 (1965).

[5] R. V. Lange, Phys. Rev. 146, 301 (1966).

[6] H. Leutwyler, Phys. Rev. D 49, 3033 (1994).

[7] H. Watanabe and H. Murayama, Phys. Rev. X 4, 031057 (2014).

[8] T. Brauner, Symmetry 2, 609 (2010).

[9] I. Frérot, P. Naldesi, and T. Roscilde, Phys. Rev. B 95, 245111 (2017).

[10] M. Srednicki, Phys. Rev. Lett. 71, 666 (1993).

[11] L. Bombelli, R. K. Koul, J. Lee, and R. D. Sorkin, Phys. Rev. D 34, 373 (1986).

[12] C. Callan and F. Wilczek, Phys. Lett. B 333, 55 (1994).

[13] P. Calabrese and J. Cardy, J. Stat. Mech. (2004) P06002.

[14] H. Casini and M. Huerta, J. Phys. A: Math. Theor. 42, 504007 (2009).

[15] J. Eisert, M. Cramer, and M. B. Plenio, Rev. Mod. Phys. 82, 277 (2010).

[16] D. Gioev and I. Klich, Phys. Rev. Lett. 96, 100503 (2006).

[17] M. M. Wolf, Phys. Rev. Lett. 96, 010404 (2006).

[18] O. A. Castro-Alvaredo and B. Doyon, Phys. Rev. Lett. 108, 120401 (2012).

[19] M. A. Metlitski and T. Grover, arXiv:1112.5166.

[20] T. Schäfer, D. Son, M. Stephanov, D. Toublan, and J. Verbaarschot, Phys. Lett. B 522, 67 (2001).

[21] J. A. Hertz, Phys. Rev. B 14, 1165 (1976).

[22] D. Pekker and C. Varma, Annu. Rev. Condens. Matter Phys. 6, 269 (2015).

[23] C. M. Varma, J. Low Temp. Phys. 126, 901 (2002).

[24] J. I. Kapusta, Phys. Rev. D 24, 426 (1981).

[25] J. Bernstein and S. Dodelson, Phys. Rev. Lett. 66, 683 (1991).

[26] L. Alvarez-Gaume, O. Loukas, D. Orlando, and S. Reffert, J. High Energy Phys. 04 (2017) 059.

[27] Y. Hama, T. Hatsuda, and S. Uchino, Phys. Rev. D 83, 125009 (2011).

[28] A. Nicolis and F. Piazza, Phys. Rev. Lett. 110, 011602 (2013).

[29] H. Watanabe, T. Brauner, and H. Murayama, Phys. Rev. Lett. 111, 021601 (2013).

[30] S. Gongyo and S. Karasawa, Phys. Rev. D 90, 085014 (2014).

[31] T. D. Cohen, Phys. Rev. Lett. 91, 222001 (2003).
[32] M. A. Metlitski, C. A. Fuertes, and S. Sachdev, Phys. Rev. B 80, 115122 (2009).

[33] M. B. Hastings, I. González, A. B. Kallin, and R. G. Melko, Phys. Rev. Lett. 104, 157201 (2010).

[34] S. Humeniuk and T. Roscilde, Phys. Rev. B 86, 235116 (2012).

[35] I. Frérot and T. Roscilde, Phys. Rev. B 92, 115129 (2015).

[36] B. Swingle and J. McGreevy, Phys. Rev. B 93, 205120 (2016).

[37] R. R. P. Singh, R. G. Melko, and J. Oitmaa, Phys. Rev. B 86, 075106 (2012).

[38] J. Helmes and S. Wessel, Phys. Rev. B 89, 245120 (2014).

[39] I. Frérot and T. Roscilde, Phys. Rev. Lett. 116, 190401 (2016).

[40] I. Peschel and V. Eisler, J. Phys. A: Math. Theor. 42, 504003 (2009).

[41] M. Greiner, O. Mandel, T. Esslinger, T. W. Hänsch, and I. Bloch, Nature (London) 415, 39 (2002).

[42] M. P. A. Fisher, P. B. Weichman, G. Grinstein, and D. S. Fisher, Phys. Rev. B 40, 546 (1989).

[43] K. Sengupta and N. Dupuis, Phys. Rev. A 71, 033629 (2005).

[44] M. Faccioli and L. Salasnich, Phys. Rev. A 99, 023614 (2019).

[45] H. Nielsen and S. Chadha, Nucl. Phys. B 105, 445 (1976).

[46] H. Watanabe and H. Murayama, Phys. Rev. Lett. 108, 251602 (2012).

[47] Y. Hidaka, Phys. Rev. Lett. 110, 091601 (2013).

[48] S. K. Pati, S. Ramasesha, and D. Sen, Phys. Rev. B 55, 8894 (1997).

[49] S. Yamamoto, T. Fukui, and T. Sakai, Eur. Phys. J. B 15, 211 (2000).

[50] A. K. Kolezhuk, H.-J. Mikeska, and S. Yamamoto, Phys. Rev. B 55, R3336(R) (1997).

[51] M. P. Hertzberg and F. Wilczek, Phys. Rev. Lett. 106, 050404 (2011).

[52] S. Gongyo, Y. Kikuchi, T. Hyodo, and T. Kunihiro, Prog. Theor. Exp. Phys. 2016, 083B01 (2016).

[53] S. Yamamoto, S. Brehmer, and H.-J. Mikeska, Phys. Rev. B 57, 13610 (1998).

[54] J. Hauschild and F. Pollmann, SciPost Phys. Lect. Notes, 5 (2018).

[55] R. Islam, R. Ma, P. M. Preiss, M. Eric Tai, A. Lukin, M. Rispoli, and M. Greiner, Nature (London) 528, 77 (2015).

[56] T. Brydges, A. Elben, P. Jurcevic, B. Vermersch, C. Maier, B. P. Lanyon, P. Zoller, R. Blatt, and C. F. Roos, Science 364, 260 (2019). 